\title{
Aplicaciones como medio e instrumento de evaluación: implementación de quizziz y kahoot en las evaluaciones de los estudiantes de contaduría pública
}

\section{Flor Marleny Gómez Reyes ${ }^{\mathrm{a}}$}

${ }^{a}$ Doctoranda en Administración y Dirección de Empresas en la Universidad Politécnica de Valencia, Magister en Gestión de Organizaciones, Especialista en NIIF y Contadora pública. flogore@doctor.upv.es

\begin{abstract}
The world of the 21st century is framed in globalization, where information flows through communications, it is inevitable to stop technological development and new ways of learning and interacting between human beings. Teaching applications have started to emerge and have become a motivation to make classes more dynamic and interactive for students. Learning requires motivation to achieve its potential.

Therefore, it will be implemented in 4 courses, which are made up of a total of 190 students, evaluations through mobile applications (quizziz and kahoot), in order to show at the end of the course if these evaluation tools cooperate with the new ways of learning theoretical courses, in students of semesters 1,5,6 and 7 of the public accounting program of a private university located in the city of Bogotá, Colombia.
\end{abstract}

Keywords: assessment instruments, Applications, App, learning.

\section{Resumen}

El mundo del siglo XXI está enmarcado en la globalización, en donde la información fluye a través de las comunicaciones, es inevitable parar el desarrollo tecnológico y las nuevas formas de aprender e interactuar entre seres humanos. Las aplicaciones docentes han empezado a surgir y se han convertido en una motivación para hacer las clases más dinámicas e interactivas para los estudiantes. El aprendizaje requiere de motivación para alcanzar las potencializades del mismo.

Por ello, se implementará en 4 cursos, que se componen de un total de 190 estudiantes, evaluaciones a través de aplicaciones móviles denominadas: quizziz y kahoot, con el fin de evidenciar al final del curso si éstas herramientas de evaluación cooperan con las nuevas formas de aprendizaje de cursos teóricos, en los estudiantes de los semestres 1, 5, 6 y 7 del programa de contaduría pública de una universidad privada ubicada en la ciudad de Bogotá, Colombia.

Palabras clave: instrumentos de evaluación, Aplicaciones, App, aprendizaje. 


\section{Introducción}

Los profesionales de la contaduría pública en Colombia, cursan asignaturas de orden práctico, teóricopráctico y teórico. En el campo de los cursos teóricos es usual que el alumnado los considere aburridos, tediosos y con bajo nivel de aplicabilidad, sin embargo, en esta investigación se pretende demostrar que esta percepción de los estudiantes sobre los cursos teóricos no es a razón del curso en sí, sino de los métodos, metodologías y herramientas que usa o deja de usar el docente en la enseñanza.

Está demostrado que el ser humano trabaja de manera más proactiva si tiene una motivación (Ausín, Abella, Delgado, \& Hortigüela, 2016). Por ello, en este documento se parte de la premisa de que el docente debe promover escenarios que propicien la motivación del estudiante en su proceso de aprendizaje. Una de las estrategias que se propone para tal fin, es que el proceso de evaluación se realice a través de la implementación de aplicaciones que puedan suplir las pruebas de papel, garantizando la misma calidad para la calificación, pero aumentando los niveles de aprendizaje en el estudiante mediante la evaluación.

Con base en lo anterior, en cuatro cursos teóricos compuestos por 190 estudiantes del programa de contaduría pública, se implementaron todas las pruebas evaluativas a través de dos aplicaciones denominadas quizziz y kahoot, con el fin de observar los cambios que generan estas apps en los escenarios del aula, los estudiantes y sus procesos de aprendizaje.

Dentro de los resultados se observa que el cambio en los medios e instrumentos de evaluación afectaron de manera positiva el proceso de evaluación del aprendizaje de los estudiantes. Este resultado se da apoyado con el ejemplo que el rol del docente causa en el alumnado y la motivación que éste genera en los estudiantes a través del cambio de instrumentos.

\section{Objetivos}

El objetivo principal es implementar en el aula de clase, nuevos instrumentos de evaluación que propendan por el desarrollo en el aprendizaje de los estudiantes de contaduría pública de una forma dinámica.

Dentro de los objetivos específicos se encuentran los siguientes:

- Aplicar las evaluaciones a los estudiantes de cada asignatura a través de aplicaciones móviles como estrategia de aprendizaje.

- Analizar las situaciones generadas en el aula de clase y en la actitud de aprendizaje de los estudiantes frente al cambio de instrumentos de evaluación.

- Evaluar la incidencia de rol del profesor en la actualización de nuevas formas en la implementación de las evaluaciones y la influencia de este proceso en el aprendizaje de los estudiantes.

\section{App como instrumento de evaluación}

\subsection{Metodología}

La metodología para la implementación de las apps como instrumentos de evaluación, comenzó con la búsqueda de las mismas, filtrando las adecuadas para estudiantes de ciencias económicas y contaduría 
pública, obteniendo como decisión, aplicar quizzis y kahoot, por características como: uso amigable para el estudiante y el profesor, gratuita y dinámica en las evaluaciones.

Las evaluaciones a través de las apps seleccionadas se aplicaron a 190 estudiantes del programa académico de contaduría pública ${ }^{1}$ de una universidad privada ubicada en la ciudad de Bogotá Colombia. Los cursos utilizados como prueba fueron los siguientes:

Tabla 1 Muestra de estudiantes y cursos donde se aplicaron evaluaciones a través de apps

\begin{tabular}{clcc}
\hline No. & \multicolumn{1}{c}{ Nombre del curso } & Semestre & Cantidad de alumnos \\
\hline 1 & Filosofía de la regulación profesional & 1 & 47 \\
2 & Escuelas del pensamiento contable & 5 & 28 \\
3 & Teorías de la contabilidad & 6 & 45 \\
4 & Métodos y metodologías de la investigación & 7 & 70 \\
& contable & & 190 \\
Total alumnos que realizaron evaluaciones a través de apps & & \\
\hline
\end{tabular}

Fuente: Elaboración propia (2020)

\section{2. innovación en los instrumentos de evaluación como estrategia didáctica para el desarrollo del aprendizaje en los estudiantes}

\subsubsection{El uso de aplicaciones como estrategia didáctica en las evaluaciones del aprendizaje}

Las docentes requieren evaluar los conocimientos adquiridos y las competencias desarrolladas por sus estudiantes (Nicolás \& Ramos, 2019), algunos las realizan de manera verbal, la gran mayoría de forma escrita, a través de preguntas abiertas o cerradas, de selección múltiple, etc. (Hamodi, Pastor, \& Pastor, 2015). Cualquiera que sea la forma de evaluar, este proceso siempre genera escenarios de ansiedad en los estudiantes (Parra-Sandoval, Rodríguez-Álvarez, Paz Rodríguez-Hopp, \& Díaz-Narváez, 2018), esta emoción es considerada para (Flores Tapia, Chávez Becerra, \& Aragón Borja, 2016) como:

un conjunto de respuestas desagradables que pueden manifestarse de manera independiente a nivel cognitivo, fisiológico y motor, de escaso control voluntario ante un estímulo o situación, ya sea interna o externa, evaluada por el individuo como potencialmente amenazante, aunque objetivamente pueda resultar no peligrosa (P. 36).

Por este motivo, cuando llega la semana de exámenes, son días que implican un grado de estrés en los estudiantes, ya que se crea un estado emocional de presión en los alumnos (Martín, 2007). Por ello, uno de los objetivos de este estudio es observar en los estudiantes, si al cambiar la herramienta de evaluación de pruebas de papel y lápiz por evaluaciones a través de sus móviles, generará en ellos un menor grado de estrés y por tanto, menos bloqueos mentales causados por los nervios y ansiedad que generan los exámenes (Flores Tapia et al., 2016). Así mismo, evidenciar que la adquisición de contenidos y competencias en los estudiantes es la misma usando las herramientas (Quizziz y Kahoot) que usando las medidas tradicionales (papel y lápiz).

Con base en lo anterior, el experimento de esta investigación consiste en usar las mismas preguntas que se encuentran diseñadas para ser entregadas en papel y transcribirlas en la aplicación (Kahoot o Quizziz). Garantizando que la calidad de la prueba sea la misma pero cambiando el medio por el cual los alumnos

\footnotetext{
${ }^{1}$ El programa de contaduría pública se compone de ocho semestres en total y su modelo pedagógico es por competencias.
} 
responderán (móviles) (Robinson, 1952); asegurando así que los estudiantes adquieran el mismo conocimiento y competencias.

Desde la primera prueba aplicada, los estudiantes manifestaron que sus niveles de estrés y ansiedad ante el examen bajaron. Estas afirmaciones se recolectaron a través de la encuesta que se realizó al final de cada curso y la cual se encuentra en el ítem de resultados de este documento. Asi mismo, se evidenció mejora en las competencias y adquisición de contenido en los estudiantes, mejorando la calificación media en cada curso.

La prueba a través de sus móviles cambió por completo el escenario de los típicos exámenes en donde todos los estudiantes están en completo silencio y nadie puede levantar la mirada de sus hojas. Esta nueva forma de realizar las evaluaciones genera en los estudiantes diversas emociones de alegría y competencia, así como, forma un ambiente de tranquilidad que les permité analizar, evaluar y recordar los conocimientos adquiridos a lo largo del curso. Estas aplicaciones son muy dinámicas ya que a medida que los estudiantes van respondiendo las preguntas, la herramienta genera reportes inmediatos sobre el avance de la prueba, en donde el alumnado puede ver proyectado sus posiciones de respuesta con respecto a los demás compañeros del grupo. Generando una competencia sana en donde al final de la prueba cada estudiante desea ver su nombre entre los primeros lugares del podio.

Con la aplicación de los exámenes a través de las apps en los móviles del alumnado, se detectó que los estudiantes estaban más activos y alertas, más emocionados y menos agobiados por la presentación de los mismos. Esta parte emocional es de vital importancia, dado que el contexto de los estudiantes del programa de contaduría pública es particular, en tanto que la gran mayoría trabaja todo el día desde las 7:00 am hasta las 5:30 pm. Posteriormente, deben desplazarse desde sus zonas de trabajo hasta la universidad, lo cual puede tardar alrededor de una hora o más, para llegar a clase de 6:00 pm hasta las 10:00 pm.

Dados los niveles de estrés y cansancio con que llegan los estudiantes a clase y más a los exámenes, las pruebas a través de las apps en sus móviles, generó escenarios dinámicos, alegres y llenos de conocimiento que promueve en los alumnnos un aprendizaje analítico, reflexivo, discursivo y no solamente memorístico.

Así mismo, la aplicación de herramientas tecnológicas motivó en los estudiantes el interés de aprendizaje sobre cursos que para ellos eran de bajo interés, dado el alto grado teórico y poco práctico. Puesto que los estudiantes de contaduría pública en su gran mayoría trabajan como auxiliares contables, consideran estos cursos como: aburridos, tediosos y poco aplicables en sus labores de trabajo. Sin embargo, al enseñar y evaluar los conocimientos de estas asignaturas a través de las aplicaciones y sus móviles, aumentó los niveles de participación y concentración en los estudiantes, dado el dinamismo que se genera en el aula de clase.

En la figura 1 se presenta el sistema de evaluación del aprendizaje en el estudiante, el cual es un ciclo que comienza con el estudiante, termina en él y vuelve a comenzar con él: 


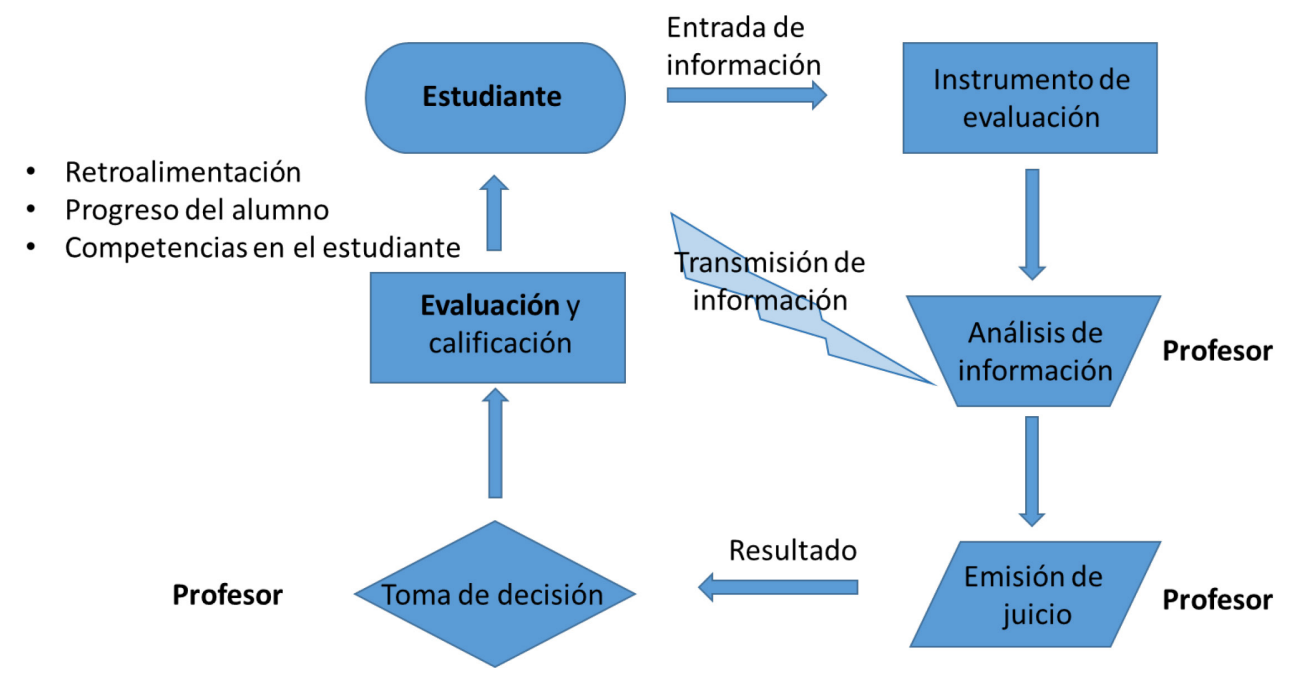

Fig. 1. sistema de evaluación del aprendizaje en el estudiante

Como se observa en la figura 1. el proceso comienza con el estudiante el cual ingresa información en un instrumento de evaluación diseñado por la institución y/o el profesor, quien realiza un proceso de análisis de la información que ha entrado y posteriormente sale el resultado del profesor a través de la emisión de un juicio, el cual es el insumo para la toma de decisión respecto de la evaluación y calificación hacia el estudiante.

En la fase final del sistema de evaluación el profesor realiza la retroalimentación al alumno a cerca de su proceso (García-Planas \& Taberna, 2019) y competencias a fortalecer; el estudiante toma esta información y la procesa en su aprendizaje, analizándola, reflexionando sobre ella y generando un proceso interno que le permitirá comenzar de nuevo en el ciclo del sistema de evaluación de su aprendizaje.

En todo el ciclo hay una constante transmisión de información entre el profesor-estudiante y enseñanzaaprendizaje (Arceo, Ramos, Almeida, \& Jeronimo, 2014) y es este proceso cíclico el que crea en el estudiante el aprendizaje. Por ello, es importante implementar herramientas e instrumentos que apoyen, desarrollen y fomenten el aprendizaje en el alumnado.

\subsection{2 el rol del profesor en la aplicación de nuevas formas de evaluar el aprendizaje de los estudiantes}

Las tendencias del mundo giran alrededor de la tecnología, es inevitable pretender pararla o vivir fuera de ella (Gasca \& Machuca, 2019). Es responsabilidad de los profesores estar actualizados en las herramientas, instrumentos y medios que afectan el desarrollo de las competencias de los futuros profesionales que están formando. Por ejemplo, en el área de contaduría pública, cada año las casas de software contable realizan mejoras y avances en los procesos contables, lo cual minimiza tareas operativas que hasta hace unos años eran del quehacer manual de un contador, analista financiero, tesorero y todo aquel que tenía funciones de archivo y procesos numéricos. Es por ello que no solo es suficiente enseñar al estudiante sobre los procesos contables, financieros y tributarios; también es necesario apoyarlos en desarrollar competencias y habilidades (Marrero, Mohamed, \& Jordi, 2018) que les permitan desempeñar su labor en organizaciones donde el contacto con la tecnología es creciente de forma exponencial (Nahón, 2007). 
Por lo anterior, pretender alejar al estudiante de móviles, computadores, tabletas y toda herramienta tecnológica, es pretender ocultarles una realidad; en cambio de ello se propone que el profesor direccione de manera positiva el uso adecuado de los nuevos medios tecnológicos de que se disponen en el siglo XXI (Duque, Santos Borja, Andrea, \& Medina, 2018).

La mejor enseñanza se da a través del ejemplo, es por ello que los docentes deben desarrollar primero sus competencias y habilidades tecnológicas para lograr implementarlas en el aula y de esta manera dar ejemplo a sus estudiantes.

\subsubsection{El ejemplo del docente como motivación en el aprendizaje del estudiante}

El ejemplo es el mejor discurso que un docente puede dar a sus estudiantes, por ello, la docente del curso de métodos y metodologías de investigación contable (curso que representa el $36.8 \%$ del total de la población objeto de estudio) participó en el mismo congreso internacional de ciencias económicas en el que instó a sus estudiantes a inscribirse, con el objeto de dar ejemplo y motivar a que el $100 \%$ de sus estudiantes se registraran en el mismo evento o buscarán un congreso de investigación contable similar y postularan sus proyectos de investigación desarrollados en clase. En la figura 2 se evidencia a la docente participando en la misma línea de investigación que sus estudiantes.

La inscripción de los estudiantes en congresos de investigación fue del 100\%, lo cual demostró que el ejemplo de la docente fue una variable fundamental. El siguiente reto era realizar trabajos de alta calidad, de tal forma que fueran aceptados en los congresos donde los estudiantes se postularon.

Ejecutando el proceso del sistema de evaluación (Fig.1) se logró que el alumnado analizara, reflexionara e indagara más allá de lo requerido por la docente. Así mismo, participara de forma proactiva en su proceso de aprendizaje y evaluación, teniendo una constante comunicación con su profesor a través de las aplicaciones tecnológicas. Todo ello aportó al compromiso del estudiante en la elaboración de un trabajo de calidad (Hamodi et al., 2015).

Teniendo la meta clara (postular sus documentos y que fueran aceptados), generó en los estudiantes una motivación que repercutió en que los mismos dedicaran tiempo extra a la elaboración de sus proyectos y por tanto necesitaron más tiempo del docente para la revisión. Por tal motivo, se utilizaron las plataformas digitales para el seguimiento y control de los avances de los proyectos, teniendo una comunicación fluida y constante entre el docente, el alumno y las retroalimentaciones de sus proyectos. Al final del semestre el $100 \%$ de los trabajos de los estudiantes postulados en congresos nacionales de investigación en la ciudad de Bogotá fueron aceptados y publicados.

Es importante resaltar que uno de los trabajos fue galardonado como el mejor proyecto en la línea de investigación denominada: "Contabilidad, gestión e innovación" en el XX Congreso Internacional de ciencias Contables, Financieras y Afines, como se observa en la figura 3. 


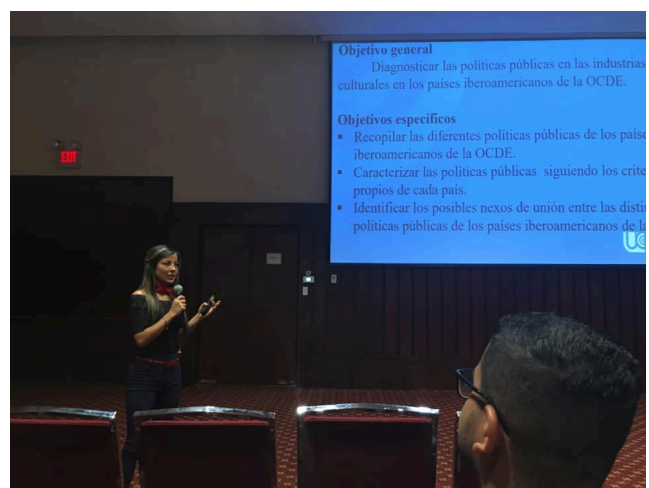

Fig. 2. profesora presentando su Proyecto en el XX Congreso Internacional de ciencias Contables, Financieras y Afines

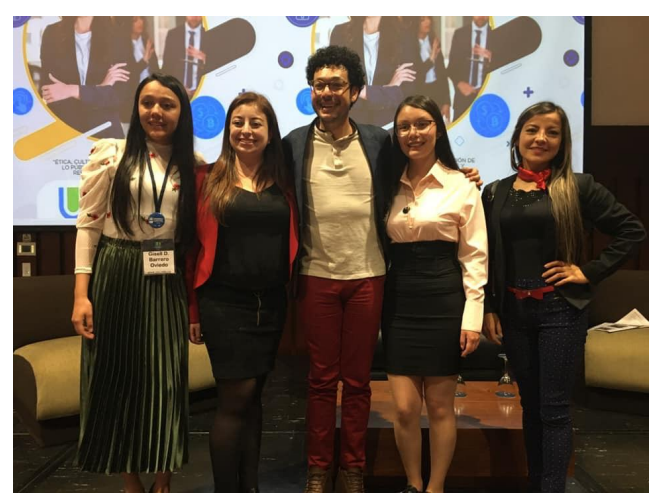

Fig. 3. Estudiantes del curso métodos y metodologías de la investigación, ganadoras con el primer puesto por su investigación

El rol del docente en la evaluación de cada proyecto con ayuda de las aplicaciones tecnológicas, se convirtió más que en un medio de calificación, en una herramienta para el seguimiento y retroalimentación del desarrollo de los proyectos de los estudiantes (Navío, Domínguez, \& Zagalaz, 2019).

El docente del siglo XXI debe ser un docente que debe estar en constante aprendizaje de las nuevas aplicaciones tecnológicas (Arceo et al., 2014), las cuales le permitan interactuar de manera efectiva con sus estudiantes, genere motivación (Villalobos-Abarca, Herrera-Acuña, Ramírez, \& Cruz, 2018) y deseos de aprendizaje contínuo en sus alumnos.

\section{Resultados}

Se pudo observar una mejora en la participación de los estudiantes en las clases, a pesar de ser cursos considerados por los estudiantes como aburridos y sin objetivo práctico, pero que, al cambiar las herramientas de evaluación, afectaron de forma positiva su aprendizaje sobre dichas asignaturas.

La motivación creada por los nuevos escenarios que se formaron en el aula de clase, debido al cambio en los instrumentos de evaluación apoyados por las aplicaciones, generaron procesos de aprendizaje en el estudiante, siendo reflejado en la calidad de los proyectos finales para cada asignatura. 
La calidad de los trabajos presentados por los estudiantes mejoró, al punto de que los proyectos del curso de métodos y metodologías de la investigación fueron presentados en congresos nacionales e internacionales de investigación de las ciencias económicas y todos fueron aceptados y publicados.

Se logró sensibilizar a los estudiantes objeto de estudio, sobre la importancia de las asignaturas teóricas como base de los procesos económicos de las empresas y de los países.

A continuación, se presentan los resultados de las respuestas de estudiantes a la encuesta realizada al final de cada curso, con el fin de plasmar la percepción del alumnado con respecto a los nuevos instrumentos de evaluación aplicados.

En la figura 4 se observa que el $100 \%$ de los estudiantes consideran que la implementación de las aplicaciones como quizziz y kahoot en los procesos de evaluación de sus aprendizajes, fueron interesantes ya que la mitad de los encuestados dijo estar totalmente de acuerdo y los restantes de acuerdo.

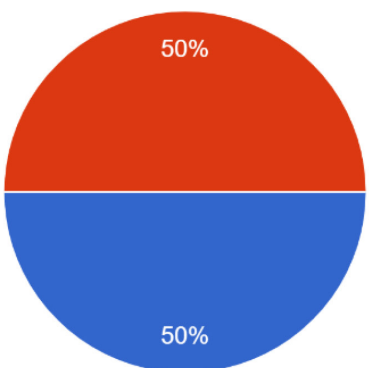

Totalmente de acuerdo

De acuerdo

Indiferente

En desacuerdo

Totalmente en desacuerdo

Fig. 4. Valoración de estudiantes a la pregunta: ¿fue interesante el empleo de una aplicación (quizziz o Kakoot) para la realización de las pruebas de conocimiento?

El $100 \%$ de los encuestados manifestó que le gustaría que sus docentes implementaran más las aplicaciones quizziz y kahoot en la realización de los exámenes y pruebas, ya que la interacción con sus móviles, tabletas, ordenadores y la interacción con sus compañeros, hace que los procesos evaluativos de sus aprendizajes se ejecuten bajo escenarios proactivos y motivadores.
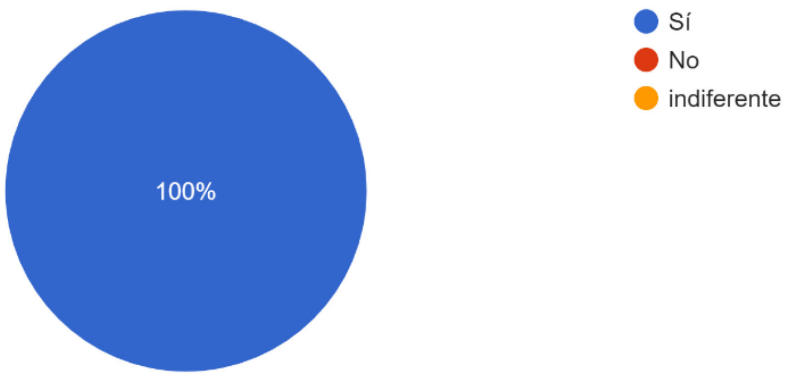

Fig. 5. Valoración de los estudiantes a la pregunta: ¿le gustaría que los docentes aplicaran más estas aplicaciones (quizziz, kahoot, entre otros) en la realización de exámenes y pruebas?

Para la figura 6. se observa que el $87.5 \%$ de los estudiantes encuestados afirma sentirse más tranquilo cuando realiza sus exámenes mediante las aplicaciones de quizziz y kahoot, en comparación con el estrés y ansiedad que experimentan cuando el escenario de la prueba es en papel, en un ambiente tenso en donde en ocasiones experimentan bloqueos mentales que no les permiten responder con claridad. 


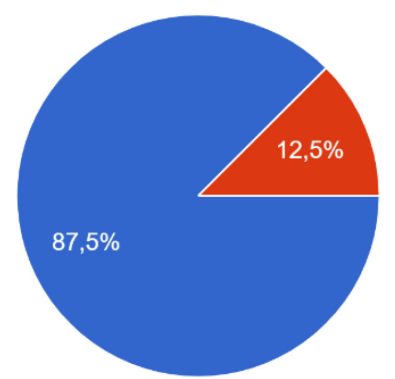

Más tranquilo que cuando la realiza en papel

se siente igual responder un test por la aplicación que en papel

Mas estresado que cuando la realiza en papel

Fig. 6. Valoración de los estudiantes a la pregunta: ¿cómo se sintió cuando realizó la prueba diagnóstica a través de su celular y no a través de una hoja y un esfero?

Uno de los resultados más significativos es que los estudiantes afirman que realizar el proceso de evaluación por medio de aplicaciones, aporta en las nuevas formas de sus aprendizajes. Estas manifestaciones de la encuesta se ven reflejadas en los resultados de los trabajos presentados por los estudiantes en el aula de clase y en escenarios externos como fueron los congresos nacionales e internacionales de investigación, lo que nos confirma que los estudiantes lograron adquirir el contenido y desarrollar las competencias que presentaba cada curso; en algunos casos los resutados superaron las expectativas (galardones a mejores investigaciones, resultado del proceso en el aula).

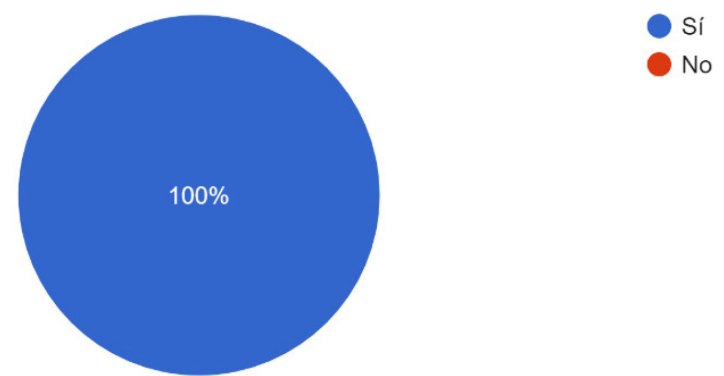

Fig. 7. Valoración de los estudiantes a la pregunta: ¿considera que las pruebas que realizó su docente a través de Quizzis y/o Kahoot, aportaron en sus nuevas formas de aprendizaje?

En la figura 8 se evidencia que el $87.5 \%$ de los estudiantes encuestados afirma que los escenarios que generan la implementación de las aplicaciones en los procesos evaluativos, son proactivos, inclusivos y motivadores para estudiar y esforzarse más en su aprendizaje. En tanto el $12.5 \%$ de los encuestados manifestó que la experiencia fue positiva y didáctica.

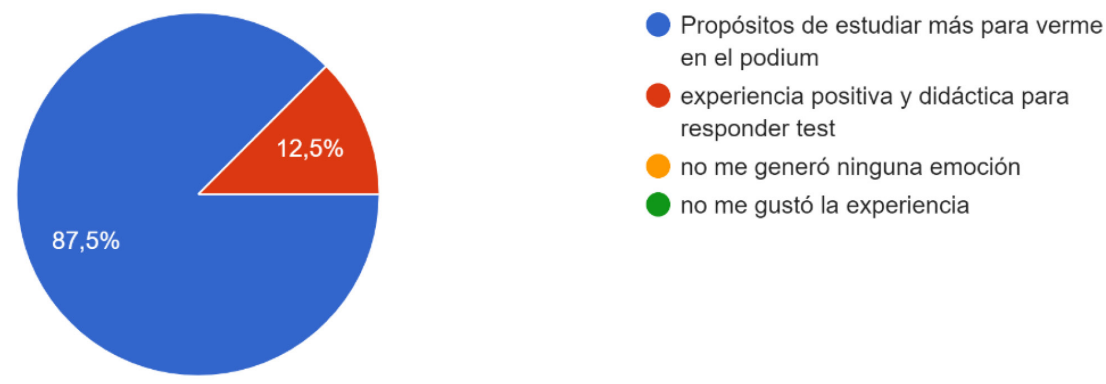

Fig. 8. Valoración de los estudiantes a la pregunta: ¿qué emoción le produjo ver en el tablero proyectado los lugares en que quedó cada compañero al terminar la prueba? 
Aplicaciones como medio e instrumento de evaluación: implementación de quizziz y kahoot en las evaluaciones de los estudiantes de contaduría pública

Finalmente, en la tabla 2 se observan algunos comentarios escritos por los estudiantes respecto a su percepción sobre la aplicación de los nuevos instrumentos de evaluación a través de sus móviles:

Tabla 2 Respuestas de algunos estudiantes a la pregunta: ¿cómo le pareció la implementación de las herramientas tecnológicas en el aula?

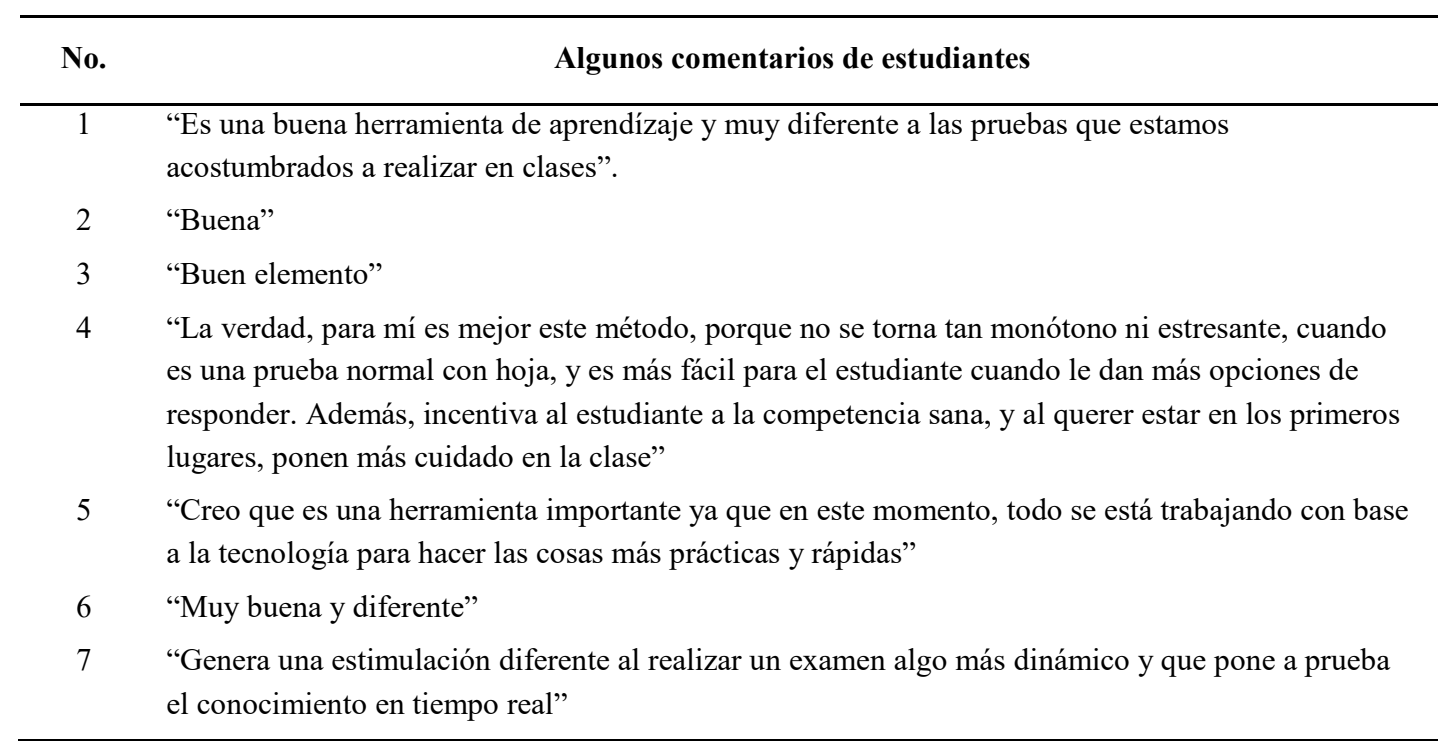

Fuente: Elaboración propia basada en encuestas (2020)

En general entre el $87 \%$ y el $100 \%$ de los estudiantes encuestados de los cursos teóricos del programa de contaduría pública, afirmaron que la implementación de las aplicaciones como quizziz y kahoot en sus evaluaciones aportó en su proceso de aprendizaje.

\section{Conclusiones}

El mundo del siglo XXI ha entrado en procesos dinámicos en donde las tecnologías son cada vez más usuales, la revolución 4.0 seguirá tomando protagonismo en todos los sectores. Es así como el sector de la educación debe estar en constante contacto en la cuarta revolución industrial. Por tanto, es responsabilidad de los docentes, que estén actualizados de los conocimientos propios del área como de las nuevas formas en que los estudiantes aprenden.

Es así que los procesos de evaluación del aprendizaje de los estudiantes, deben ser compatibles con las nuevas formas en que el alumnado interactúa con el entorno personal, laboral y académico.

El rol del docente es uno de los ejes del sistema de evaluación, pero el principal engranaje del sistema es el estudiante; quien debe ser cada vez más proactivo en su aprendizaje, adquiriendo así habilidades y competencias, las cuales no solo se adquieren en los cursos prácticos, sino también en los teóricos, siendo estos últimos el fundamento teórico, analítico y reflexivo de las áreas del conocimiento. Pero es labor del docente general los escenarios pedagógicos que propendan por la motivación del estudiante.

Por lo anterior, los docentes deben romper los paradigmas en los procesos de evaluación, incluyendo más aplicaciones y herramientas tecnológicas que aporten en el aprendizaje de los estudiantes.

Las conclusiones de este documento se realizaron en el marco del confinamiento de la pandemia COVID19 en donde se puede evidenciar que el sector de la educación está apoyando sus procesos académicos a 
través de la tecnología, las redes y todo lo que implica la revolución 4.0. Por tanto, es una necesidad que tanto docentes como estudiantes, adquieran competencias y habilidades tecnológicas, ajustando los procesos de evaluación del aprendizaje a las nuevas dinámicas de un mundo globalizado y cambiante.

\section{Referencias}

Arceo, G., Ramos, E., Almeida, M., \& Jeronimo, R. (2014). Análisis de la gestión del conocimiento y las tecnologias de informacion en el Ámbito docente universitario. Congreso Iberoamericano de Ciencia, Tecnología, Innovación y Educación, 1521, 1-18.

Ausín, V., Abella, V., Delgado, V., \& Hortigüela, D. (2016). Aprendizaje Basado en Proyectos a través de las TIC. Una Experiencia de Innovación Docente desde las Aulas Universitarias Project-Based Learning through ICT. An Experience of Teaching Innovation from University Classrooms. Publicado Formación Universitaria, 9(3), 31-38. https://doi.org/10.4067/S0718-50062016000300005

Duque, A. F., Santos Borja, D. E., Andrea, Y., \& Medina, T. (2018). Development of skills for the fourth revolution industrialization through learning methodologies based on problems and projects. Innovation in Education and Inclusion, (1). https://doi.org/10.18687/LACCEI2018.1.1.446

Flores Tapia, M. de los Á., Chávez Becerra, M., \& Aragón Borja, L. E. (2016). Situaciones que generan ansiedad en estudiantes de Odontología. Journal of Behavior, Health \& Social Issues, 8(2), 35-41. https://doi.org/10.1016/j.jbhsi.2016.11.004

García-Planas, I. M. I., \& Taberna, J. (2019). la utilización de pbl para la evaluación de la competencia de sostenibilidad y compromiso social en estudios de ingeniería.

Gasca, G., \& Machuca, L. (2019). la era de la cuarta revolución industrial. Revista Ibérica de Sistemas y Tecnologías de Información., 34.

Hamodi, C., Pastor, V. M. L., \& Pastor, A. T. L. (2015). Medios, técnicas e instrumentos de evaluación formativa y compartida del aprendizaje en educación superior. Perfiles Educativos, 37(147), 146-161. https://doi.org/10.1016/j.pe.2015.10.004

Marrero, O., Mohamed, R., \& Jordi, X. (2018). Soft Skills: Necessary for the Integral Training of the University Student. Revista Cientifica ECOCIENCIA, 1-19.

Martín, I. M. (2007). Estrés académico en estudiantes universitarios Isabel. Apuntes de Psicología, 25(1), 87-99.

Nahón, A. E. (2007). Redefinición del en red "en la cuarta revolución industrial. Apertura, 10, 149-163.

Navío, E. P., Domínguez, M. M., \& Zagalaz, J. C. (2019). Perception of the professional competences of last year's students of pre-primary education and primary education degrees and students of training teachers master. Journal of New Approaches in Educational Research, 8(1), 58-65. https://doi.org/10.7821/naer.2019.1.344

Nicolás, A. M. B., \& Ramos, P. R. (2019). Investigación-acción y aprendizaje basado en proyectos: Una revisión bibliográfica. Perfiles Educativos, 40(163), 109-122.

Parra-Sandoval, J. M., Rodríguez-Álvarez, D., Paz Rodríguez-Hopp, M., \& Díaz-Narváez, V. (2018). Relación entre estrés estudiantil y reprobación Relationship between student stress and reprobation. Barranquilla (Col.), 34(1), 47-57. Retrieved from https://orcid.org/0000-0002-

Robinson, B. Y. C. (1952). Persia, the keystone: Anglo-Persian relations since 1946. The Round Table, 43(169-172), 28-40. https://doi.org/10.1080/00358535208451787

Villalobos-Abarca, M. A., Herrera-Acuña, R. A., Ramírez, I. G., \& Cruz, X. C. (2018). Aprendizaje Basado en Proyectos Reales Aplicado a la Formación del Ingeniero de Software. Formación Universitaria, 11(3), 97112. https://doi.org/10.4067/S0718-50062018000300097 